\title{
ON THE CHERN CLASSES AND THE EULER CHARACTERISTIC FOR NONSINGULAR COMPLETE INTERSECTIONS
}

\author{
VICENTE NAVARRO AZNAR
}

\begin{abstract}
AbSTRACr. It is shown that Hirzebruch's result on the Chern classes of a complete intersection of nonsingular hypersurfaces in general position in $\mathbf{P}_{N}(\mathbf{C})$, is also valid for any nonsingular complete intersection. Then relations between Euler characteristic, class and Milnor number are pointed out.
\end{abstract}

1. Introduction. Let $\mathbf{P}_{N}(\mathbf{C})$ be an $N$-dimensional complex projective space. An $n$-dimensional algebraic subvariety $V$ of $\mathbf{P}_{N}(C)$ is called a complete intersection if the homogeneous ideal of $V$ in $\mathbf{C}\left[z_{0}, \ldots, z_{N}\right]$ is generated by $p=N-n$ homogeneous polynomials, $f_{1}, \ldots, f_{p}$. So, if $V_{i}=\operatorname{loc}\left[f_{i}\right]$, we have $V=V_{1} \cap \ldots \cap V_{p}$.

It is well known that if the $V_{i}$ are nonsingular and are in general position, then $V$ is nonsingular too; in this case the Chern classes of $V$ can be obtained in terms of the degrees of $V_{1}, \ldots, V_{p}$ (see [11]). Nevertheless, examples are easily found in which, though $V$ is nonsingular, it is not possible to find generators $f_{i}$ of the homogeneous ideal of $V$ that would give nonsingular $V_{i}$, $1<i<p$. This is, for example, the case of the sextic

$$
z_{0}^{3}+z_{1}^{3}+z_{2}^{3}=0, \quad z_{1}^{2}+z_{2}^{2}+z_{3}^{2}=0
$$

in the space $\mathbf{P}_{3}(\mathbf{C})$.

In $\$ 2$ we prove that it is not necessary to assume that the hypersurfaces $V_{i}$ are nonsingular and in general position, since the expression of the Chern classes of $V$ in terms of the degrees of the $V_{i}$ is valid even if we only assume that $V$ is nonsingular.

In $\$ 3$ we give an elementary proof for the formula giving the Euler characteristic of a nonsingular complete intersection that does not use its relation with the top Chern class, while in $\$ 4$ we give two applications of this formula, obtaining the class of a nonsingular complete intersection and the Milnor number of a germ of a homogeneous complete intersection with an isolated singularity.

2. The Chern classes. The result for which we are aiming will be obtained from the following.

Received by the editors November 27, 1978.

AMS (MOS) subject classifications (1970). Primary 57D20, 32B10.

Key words and phrases. Chern classes, Euler characteristic, class, nonsingular complete intersection, Milnor number, germs. 
LEMMA. If $V$ is an n-dimensional nonsingular complete intersection in $\mathbf{P}_{\boldsymbol{N}}(\mathbf{C})$, then there is a system of generators $f_{1}, \ldots, f_{p}$ of the homogeneous ideal of $V$, with $p=N-n$, so that the varieties

$$
W_{i}=\operatorname{loc}\left[f_{1}, \ldots, f_{i}\right], \quad 1 \leqslant i \leqslant p
$$

are nonsingular.

Proof. Let $g_{1}, \ldots, g_{p}$ be an arbitrary system of generators of id( $\left.V\right)$ and suppose that $d_{1} \geqslant d_{2} \geqslant \cdots \geqslant d_{p}$, where $d_{i}=\operatorname{deg} g_{i}, 1 \leqslant i \leqslant p$. Then, if we consider the linear system of divisors of degree $d_{1}$ defined by

$$
\lambda_{1} g_{1}+\sum_{\substack{1<i<p \\ 0<j<N}} \lambda_{i j} z_{j}^{d_{1}-d_{i}} g_{i},
$$

we can deduce, from the parametric transversality theorem in [9] (essentially the Sard theorem), that there are constants $\lambda_{i j}$ so that if

$$
f_{1}=g_{1}+\sum \lambda_{i j} z_{j}^{d_{1}-d_{i}} g_{i}
$$

then $W_{1}=\operatorname{loc}\left[f_{1}\right]$ is nonsingular. Suppose now that we have already found $f_{1}, \ldots, f_{k}$, where $k \geqslant 1$, so that $\left[f_{1}, \ldots, f_{k}, g_{k+1}, \ldots, g_{p}\right]=\mathrm{id}(V)$ and $W_{j}=$ $\operatorname{loc}\left[f_{1}, \ldots, f_{j}\right]$ is nonsingular if $1 \leqslant j \leqslant k$. Considering the linear system on $W_{k}$ defined by

$$
\mu_{1} g_{k+1}+\sum_{\substack{k<i<p \\ 0<j<N}} \mu_{i j} z_{j}^{d_{k+1}-d_{i}} g_{i}
$$

we deduce, again from the parametric transversality theorem, that there are constants $\mu_{i j}$ so that if

$$
f_{k+1}=g_{k+1}+\sum \mu_{i j} z_{j}^{d_{k+1}-d_{i}} g_{i}
$$

then $W_{k+1}=\operatorname{loc}\left[f_{1}, \ldots, f_{k+1}\right]$ is nonsingular and obviously $\left[f_{1}, \ldots, f_{k+1}, g_{k+2}, \ldots, g_{p}\right]=\operatorname{id}(V)$.

Let $H^{d}$ be a line bundle over $\mathbf{P}_{N}(C)$ defined by the linear system of hypersurfaces of degree $d \geqslant 1$ in $\mathbf{P}_{N}(\mathbf{C})$. It is well known that $H^{d}$ has $c_{1}\left(H^{d}\right)=d h \in H^{2}\left(\mathbf{P}_{N}(\mathbf{C}), \mathbf{Z}\right)$, where $h$ is a generator of $H^{2}\left(\mathbf{P}_{N}(\mathbf{C}), \mathbf{Z}\right)$.

Let $V$ be a nonsingular complete intersection in $\mathbf{P}_{N}(C)$. We shall say that $V$ has degrees $\left(d_{1}, \ldots, d_{p}\right)$ if $\operatorname{id}(V)=\left[f_{1}, \ldots, f_{p}\right]$ and $d_{i}=\operatorname{deg} f_{i}, 1 \leqslant i \leqslant p$. If $j: V \rightarrow \mathbf{P}_{N}(\mathbf{C})$ is the embedding of $V$ in $\mathbf{P}_{N}(\mathbf{C})$, we shall write $\tilde{h}$ for $j^{*} h$.

TheOREM 1. Let $V$ be a nonsingular complete intersection in $\mathbf{P}_{N}(\mathbf{C})$ of degrees $\left(d_{1}, \ldots, d_{p}\right)$, the total Chern class of $V$ is given by

$$
c(V)=(1+\tilde{h})^{N+1}\left(1+d_{1} \tilde{h}\right)^{-1} \cdots\left(1+d_{p} \tilde{h}\right)^{-1} .
$$

Proof. The proof follows from the previous lemma and from [11, 4.8.1] by induction, as in [11].

REMARK. Applying the previous lemma, the other results of [10] or [11] about complete intersections of nonsingular hypersurfaces in general position are likewise valid for any nonsingular complete intersection. 
3. The Euler characteristic. The following well-known lemma plays an important role in the development of this section.

LEMMA. Let $V$ and $W$ be two $n$-dimensional nonsingular complete intersections in $\mathbf{P}_{N}(\mathbf{C})$ of the same degrees $\left(d_{1}, \ldots, d_{p}\right)$. Then $V$ and $W$ are diffeomorphic manifolds.

Since all the $n$-dimensional nonsingular complete intersections in $\mathbf{P}_{N}(\mathbf{C})$ of degrees $\left(d_{1}, \ldots, d_{p}\right)$ have, according to the above lemma, the same Euler characteristic, we will write it as $\chi\left(d_{1}, \ldots, d_{p} ; n\right)$; hence we can choose a particularly simple complete intersection, so that:

LEMMA. If $d_{1}, \ldots, d_{p}$ are integers $\geqslant 1$, for almost any $\left(a_{i j}\right), 1<j<p$, $j-1<i<N, a_{i j} \in \mathbf{C}$, there is a nonsingular complete intersection $V$ in $\mathbf{P}_{N}(\mathbf{C})$ of degrees $\left(d_{1}, \ldots, d_{p}\right)$, with ideal

$$
\operatorname{id}(V)=\left[\left\{\sum_{i=j-1}^{N} a_{i j} z_{i}^{d j} ; 1 \leqslant j \leqslant p\right\}\right]
$$

and such that $V \cap\left\{z_{0}=0\right\}$ is nonsingular.

Proof. It is again a simple application of the parametric transversality theorem, since, if we consider the mappings

$$
\begin{gathered}
F_{1}: \mathbf{C}^{\nu} \rightarrow C^{\infty}\left(\mathbf{C}^{N+1}-\{0\}, \mathbf{C}^{p}\right), \\
\left(a_{i j}\right) \rightarrow f\left(z_{0}, \ldots, z_{N}\right)=\left(\sum_{i=0}^{N} a_{i 1} z_{i}^{d_{1}}, \ldots, \sum_{i=p-1}^{N} a_{i p} z_{i}^{d_{p}}\right),
\end{gathered}
$$

and

$$
\begin{gathered}
F_{2}: \mathbf{C}^{\nu} \rightarrow C^{\infty}\left(\mathbf{C}^{N}-\{0\}, \mathbf{C}^{p}\right), \\
\left(a_{i j}\right) \rightarrow g\left(z_{1}, \ldots, z_{N}\right)=\left(\sum_{i=1}^{N} \dot{a}_{i 1} z_{i}^{d_{1}}, \ldots, \sum_{i=p-1}^{N} a_{i p} z_{i}^{d_{p}}\right),
\end{gathered}
$$

we immediately verify that $\phi\left(F_{1},\{0\}\right)$ and $\phi\left(F_{2},\{0\}\right)$ are open dense sets so that their intersection will also be dense.

THEOREM 2. With the same notations as above,

$$
\chi\left(d_{1}, \ldots, d_{p} ; n\right)=d_{1} d_{2} \cdots d_{p}\left\{\sum_{i=0}^{n}(-1)^{n-i}\left(\begin{array}{c}
N+1 \\
i
\end{array}\right) A_{p}^{n-i}\right\}
$$

where

$$
A_{p}^{k}=\sum_{|\alpha|=k} d_{1}^{\alpha_{1}} d_{2}^{\alpha_{2}} \cdots d_{p}^{\alpha_{p}}
$$

Proof. Let $W=\left\{z \in \mathbf{P}_{N-1} ; \sum_{i=j-1}^{N} a_{i j} z_{i}^{d_{j}}=0,2<j<p\right\}, V_{0}=V \cap\left\{z_{0}\right.$ $=0\}$ and $V_{a}=V-V_{0}$. The map $f: V_{a} \rightarrow W-V_{0}$ defined by

$$
f\left(\left[z_{0}, \ldots, z_{N}\right]\right)=\left[z_{1}, \ldots, z_{N}\right]
$$

is a $d_{1}$-sheeted covering projection, therefore $\chi\left(V_{a}\right)=d_{1} \chi\left(W-V_{0}\right)$. But 
according to the Lefschetz duality theorem, we have

$$
\chi\left(W-V_{0}\right)=\chi\left(W, V_{0}\right)=\chi(W)-\chi\left(V_{0}\right)
$$

and also

$$
\chi(V)=\chi\left(V_{a}\right)+\chi\left(V_{0}\right)
$$

therefore

$$
\chi(V)=d_{1} \chi(W)-\left(d_{1}-1\right) \chi\left(V_{0}\right)
$$

We thus obtain the induction formula

$$
\chi\left(d_{1}, \ldots, d_{p} ; n\right)=d_{1} \chi\left(d_{2}, \ldots, d_{p} ; n\right)-\left(d_{1}-1\right) \chi\left(d_{1}, \ldots, d_{p} ; n-1\right)
$$

from which the theorem follows easily, starting from the initial values $\chi\left(\mathbf{P}_{n}(\mathbf{C})\right)=n+1$ and $\chi\left(d_{1}, \ldots, d_{p} ; 0\right)=d_{1} d_{2} \cdots d_{p}$.

REMARK. A noteworthy consequence of the previous theorem is that the results contained in [2], [3], [4] and [5] are also valid for any nonsingular complete intersection.

4. Some applications. We can now apply the anterior result in order to obtain:

THEOREM 3 (Compare with [14]). If $V$ is an n-dimensional nonsingular complete intersection in $\mathbf{P}_{N}(\mathbf{C})$ of degrees $\left(d_{1}, \ldots, d_{p}\right)$, its class is given by

$$
\rho_{n}\left(d_{1}, \ldots, d_{p} ; n\right)=d_{1} d_{2} \cdots d_{p}\left\{\sum_{i=0}^{n}(-1)^{i}\left(\begin{array}{c}
N-1 \\
i
\end{array}\right) A_{p}^{n-i}\right\} .
$$

Proof. It is known (see [12]) that the dual variety $V^{*}$ of a nonsingular complete intersection $V$ in $\mathbf{P}_{N}(C)$ is a hypersurface in the dual projective space $\mathbf{P}_{N}^{*}(\mathbf{C})$ of the hyperplanes; therefore the class of $V$, i.e. the degree of $V^{*}$, will be obtained as the intersection number of $V^{*}$ with a general line of $\mathbf{P}_{N}^{*}(\mathbf{C})$. But if we interpret this line as a pencil of hyperplanes in $\mathbf{P}_{N}(\mathbf{C})$ and apply the Zeuthen-Segre formula in [1], we have

$$
\chi(V)=2 \chi\left(V \cap H_{0}\right)-\chi\left(V \cap H_{0} \cap H_{1}\right)+(-1)^{n} \rho_{n}(V)
$$

where $H_{0}$ and $H_{1}$ are two generic hyperplanes of the pencil, from which it follows:

$$
\begin{array}{r}
\rho_{n}(V)=(-1)^{n}\left\{\chi\left(d_{1}, \ldots, d_{p} ; n\right)-2 \chi\left(d_{1}, \ldots, d_{p} ; n-1\right)\right. \\
\left.+\chi\left(d_{1}, \ldots, d_{p} ; n-2\right)\right\}
\end{array}
$$

and applying Theorem 2, we obtain the expression for which we were aiming.

Finally, we apply Theorem 2 to obtain the Milnor number of the germs of homogeneous complete intersection with an isolated singularity.

Let $(X, 0)$ be an $n$-dimensional germ of complete intersection in $\mathbf{C}^{N}$, defined in a neighborhood $U$ of 0 by the functions $f_{1}, \ldots, f_{p}$; we shall say that $(X, 0)$ is an homogeneous complete intersection of degrees $\left(d_{1}, \ldots, d_{p}\right)$ if $f_{i}$ is an homogeneous polynomial of degree $d_{i}, 1 \leqslant i \leqslant p$. From this it follows immediately that in this case we can take all $C^{N}$ as $U$. We assume now that 
$(X, 0)$ has an isolated singularity, and we denote by $f: \mathbf{C}^{N} \rightarrow \mathbf{C}^{p}$ the mapping with components $f_{1}, \ldots, f_{p}$; the affine manifold $f^{-1}(y)$, where $y$ is a regular value of $f$, is called the Milnor fibre of $(X, 0)$ (see [13] and [8]). From [8] we thus know that $f^{-1}(y)$ has the homotopy type of a bouquet of $n$-dimensional spheres; the number of spheres in this bouquet is called the Milnor number of $(X, 0), \mu(X, 0)$. The following result is originally stated in [7] (see also [6]).

THEOREM 4. If $(X, 0)$ is an $n$-dimensional homogeneous complete intersection in $\mathbf{C}^{N}$, of degrees $\left(d_{1}, \ldots, d_{p}\right)$ with an isolated singularity, we have

$$
\mu(X, 0)=(-1)^{n+1}+d_{1} d_{2} \cdots d_{p}\left\{\sum_{i=0}^{n}(-1)^{i}\left(\begin{array}{c}
N \\
i
\end{array}\right) A_{p}^{n-i}\right\}
$$

Proof. Let $F=f^{-1}(y)$ be the Milnor fibre. Using a change of coordinates in $\mathbf{C}^{p}$, if necessary, we may assume that $y=(1,1, \ldots, 1)$. Since $f$ is homogeneous, we may define the projective variety $V$ of $\mathbf{P}_{N}(C)$ as

$$
V=\left\{z \in \mathbf{P}_{N}(\mathbf{C}) ; f_{i}\left(z_{1}, \ldots, z_{N}\right)-z_{0}^{d_{i}}=0,1 \leqslant i \leqslant p\right\}
$$

so $V$ is a nonsingular complete intersection of degrees $\left(d_{1}, \ldots, d_{p}\right)$ and, if $V_{0}=V \cap\left\{z_{0}=0\right\}$, we shall get $F=V-V_{0}$. But since $V_{0}$ is a complete nonsingular intersection in $\mathbf{P}_{N-1}(\mathbf{C})$, of degrees $\left(d_{1}, \ldots, d_{p}\right)$, we shall have

$$
\chi(F)=\chi\left(V, V_{0}\right)=\chi(V)-\chi\left(V_{0}\right)
$$

i.e.

$$
1+(-1)^{n} \mu=\chi(F)=\chi\left(d_{1}, \ldots, d_{p} ; n\right)-\chi\left(d_{1}, \ldots, d_{p} ; n-1\right) .
$$

Now applying Theorem 2, we have the formula we sought to obtain.

\section{BIBLIOGRAPHY}

1. A. Andreotti and T. Frankel, The second Lefschetz Theorem on hyperplane sections, Global Analysis (Papers in Honor of K. Kodaira), Univ. Tokyo Press, Tokyo, 1969, pp. 1-20.

2. B. Y. Chen and K. Oguie, Some implications of the Euler-Poincare characteristic for complete intersection manifolds, Proc. Amer. Math. Soc. 44 (1974), 1-8.

3. Complete intersection manifolds with extremal Euler-Poincaré characteristics, Proc. Amer. Math. Soc. 50 (1975), 121-126.

4. B. Y. Chen, Euler characteristics and codimension of complete intersections, Proc. Amer. Math. Soc. 71 (1978), 13-14.

5. J. Ewing and S. Moolgavkar, Euler characteristics of complete intersections, Proc. Amer. Math. Soc. 56 (1976), 390-391.

6. M. Giusti, Sur les singularités isolées d'intersections completes quasi-homogènes, Ann. Inst. Fourier (Grenoble) 27 (1977), 163-192.

7. G. M. Greuel and H. A. Hamm, Invariaten quasi-homogener vollstrandiger Durchschnitten, Invent. Math. 49 (1978), 67-86.

8. H. A. Hamm, Lokale topologische Eigenschaften komplexer Raume, Math. Ann. 191 (1971), 235-252.

9. M. W. Hirsch, Differential topology, Graduate Texts in Math., No. 33, Springer-Verlag, Berlin and New York, 1976.

10. F. Hirzebruch, Der Satz von Riemann-Roch in Faisceau-theoretischer Formulierung: einige Anwendungen und offene Fragen, Proc. Internat. Congress Mathematicians, Amsterdam, 1954, vol. III, Noordhoff, Groningen; North-Holland, Amsterdam, 1956, pp. 457-473. 
11. Topological methods in algebraic geometry, 3rd ed., Grundlehren 131, SpringerVerlag, New York, 1966.

12. S. Kleiman, The enumerative theory of singularities. Real and complex singularities, Oslo, 1976, Sijthoff \& Noordhoff, Alphen aan den Rijn, 1977, pp. 297-396.

13. J. Milnor, Singular points of complex hypersurfaces, Ann. of Math. Studies, No. 61, Princeton Univ. Press, Princeton, N.J.; Univ. Tokyo Press, Tokyo, 1968.

14. F. Severi, Sulle intersezioni delle varietá algebriche e sopra $i$ loro caratteri e singolarita proiettive, Mem. Accad. Sci. Torino Cl. Sci. Fis. Mat. Natur. 52 (1902), 61-118.

Departamento de Matematicas, etsil, Universidad Politecnica de Barcelona, DiagoNAL, 647, BARCELONA (14), SPAIN 